\title{
DOES MYOFASCIAL RELEASE TECHNIQUE CONTRIBUTE TO CERVICAL RADICULOPATHY TREATMENT? CUES FROM A NON- CONTROLLED EXPERIMENTAL DESIGN STUDY
}

\author{
Ritika Sambyal*, Monika Moitra**, Asir John Samuel***, Senthil Paramasivam Kumar**** \\ Autor correspondente: Asir John Samuel - asirjohnsamuel@mmumullana.org \\ * Post Graduate Student, Maharishi Markandeshwar Institute of Physiotherapy and Rehabilitation, Maharishi \\ Markandeshwar University, Mullana-133207. Haryana, India \\ ** Professor, Maharishi Markandeshwar Institute of Physiotherapy and Rehabilitation, Maharishi Markandeshwar University, \\ Mullana-133207. Haryana, India \\ *** BSc (Psychology), BPT, MPT(Neurosciences and Pediatric neurology), DYScEd, (PhD), Assistant Professor, Maharishi \\ Markandeshwar Institute of Physiotherapy and Rehabilitation, Maharishi Markandeshwar University, Mullana-133207. \\ Haryana, India \\ **** Professor and Head, Department of Physiotherapy, School of Allied Health Sciences, Sharda University, Greater Noida \\ - 201306. Uttar Pradesh, India
}

\begin{abstract}
Background: Neck is common and its incidence is increasing throughout the world. Neck pain with Cervical Radiculopathy (CR), nowadays, is frequently diagnosed in physiotherapy clinics and hospitals. The focus of all the existing treatments of $C R$ focuses only on relieving pain and treating the radicular symptoms rather than on the muscle dysfunction itself. Objective of study: To evaluate the effect of Myofascial Release (MFR) in decreasing Neck Disability (NDI) in patients with CR. Methods: A total of 15 patients with CR and Myofascial Trigger Points (MTrPs) in upper trapezius were recruited for the study. The intervention took place in 5 consecutive days. Data were analysed by using Wilcoxon Signed Rank Test and central tendency as mean with a 95\% Confidence Interval $(\mathrm{Cl})$. Results: After 5 days of intervention the results showed significant changes in scores of NDI 22.66 (17.3-27.9) (mean with 95\% $\mathrm{Cl} ; \mathrm{p}<0.001$ ), Numeric Pain Rating Scale (NPRS) 3.8 (3.2-4.3) (mean with $95 \% \mathrm{Cl} ; \mathrm{p}<0.001$ ) and Upper Limb Neurodynamic Test for median nerve (ULNT1) 61.25 (45.2-77.2) (mean with 95\% Cl; $p<0.001$ ). Conclusion: MFR was effective in treating the patients with $C R$.
\end{abstract}

Keywords: Manual therapy; Myofascial release therapy; Neck pain; Neck related arm pain; Rehabilitation. 


\section{INTRODUCTION}

The annual prevalence of cervical radiculopathy is 83.2 per 100,000 population. ${ }^{(1,2)}$ Cervical Radiculopathy $(C R)$ is defined as a neurologic condition that occurs because of compression and inflammation of cervical nerve root or roots in the neural foramen due to cervical disc herniation, cervical spondylosis or osteophytosis. ${ }^{(1,3)}$ Clinically, CR presents itself as a severe neck pain and a sharp or radiating pain with a tingling or burning sensation along the dermatomal distribution in upper extremity which is aggravated by neck movement and may cause symptoms like reflex loss, dermatomal numbness or myotomal weakness. ${ }^{(4-7)}$

Studies have concluded that myofascial trigger points (MTrPs) are predominantly present on the side of radiculopathy. ${ }^{(8-11)}$ MFR is considered to be effective in treating the MTrPs. ${ }^{(2-17)} A$ study has been done to assess the effect of gross MFR of upper limb and neck on pain and function in subjects with mechanical neck pain with upper limb radiculopathy which concluded that gross MFR is effective in reducing mechanical neck pain and in improving functional abilities. ${ }^{(15)}$

The patients with CR have multiple MTrPs with predominance to the side of radiculopathy. ${ }^{(8,9,11)}$ Although many interventions are accepted for $C R,{ }^{(2,16}$ ${ }^{20)}$ substantial evidence regarding effect of MFR on treating MTrPs in patients with CR is still lacking.

\section{METHODOLOGY}

This was a non-controlled experimental study design for which ethical clearance was obtained from the institutional research ethics committee. The study was conducted at inpatient department of tertiary care teaching hospital.

A total of 15 subjects were recruited for the study following criteria based on purposive sampling, a non-random sampling technique. Inclusion criteria were age between 25-45 years, either males or females, unilateral pain radiation to the dominant arm, trigger points in upper trapezius, patients showing positive cervical foraminal compression test (spurling test), manual cervical distraction test, ipsilateral limitation of cervical rotation, Upper Limb Neurodynamic Test for median nerve (ULNT1) and arm pain on Numeric Pain Rating Scale (NPRS) 4-8. The patients were excluded in presence of any of the following conditions cervical instability, vertebral artery insufficiency, spine cord compression, spine infections, previous spinal injury, recent motor vehicle accident involving cervical spine, systemic disease, severe osteoporosis and history of psychological or physical illness. The primary outcome measure was NDI and secondary outcome measures were divided into subjective which was taken as NPRS and objective which was taken as ULNT1. Written informed consents were provided by the recruited subjects before the begining of the study. After the baseline measurements of NDI, NPRS and ULNT 1, MFR was composed of gross stretch of upper quarter (arm pull) followed by gross and focused stretch were given to the upper trapezius for 5 consecutive days. Post analysis of the above outcome measures was done on the $5^{\text {th }}$ day following the intervention. Study flowchart is displayed in figure 1. 


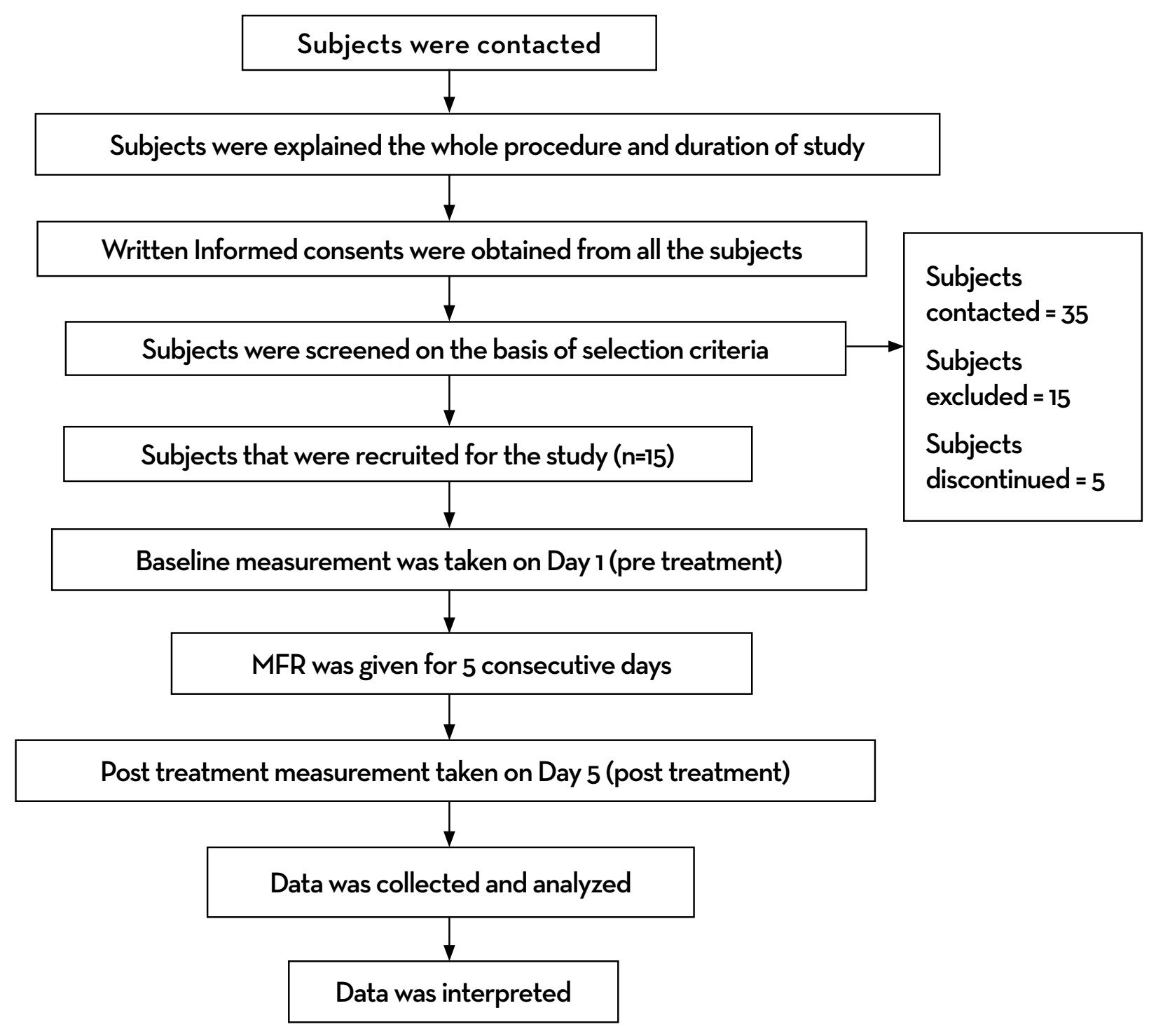

Figure 1 - Non-controlled experimental study flowchart

\section{TECHNIQUE}

In arm pull, long lever of an extremity was used to release the muscles or myofascial units. In gross stretch of the upper trapezius one hand acted as the anchor from which stretch originated and the other hand provided the stretching force. Focused MFR was given over the small segment of the upper trapezius with two fingers reinforcing each other and then a downward vertical stretch was executed. The stretch was applied to take the available slack. The hold was executed for 90-120 seconds, depending upon the release and stretch was then applied again. The sequence was repeated until the end feel was reached. The whole MFR technique is illustrated in the figures 2 to 7 . 


\subsection{ARM PULL}

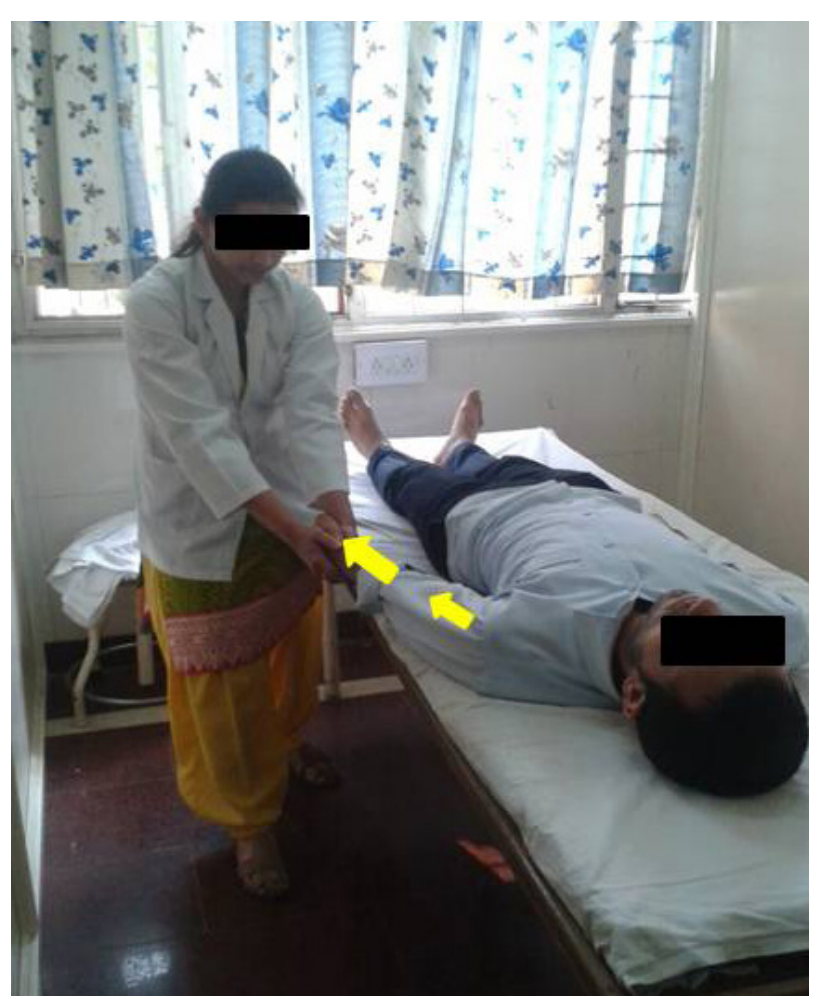

Figure 2 - Upper limb traction given by therapist using fingers and thenar eminence

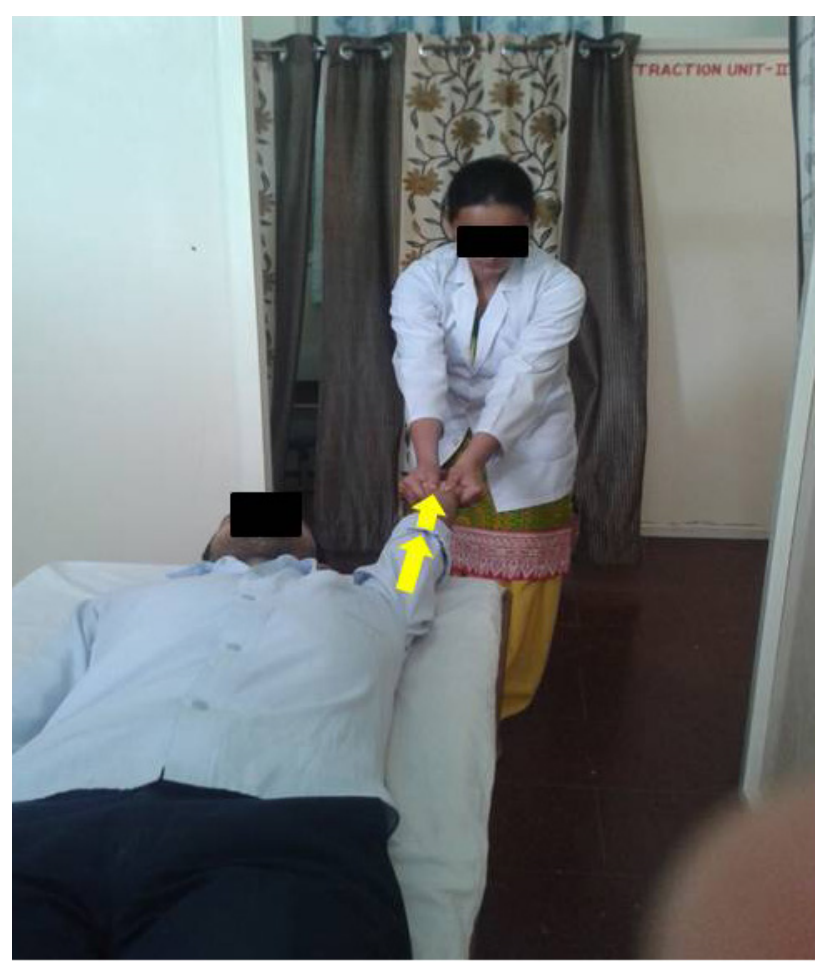

Figure 4 - Further abduction followed by flexion and internal rotation to take patient's arm proximal to his ear

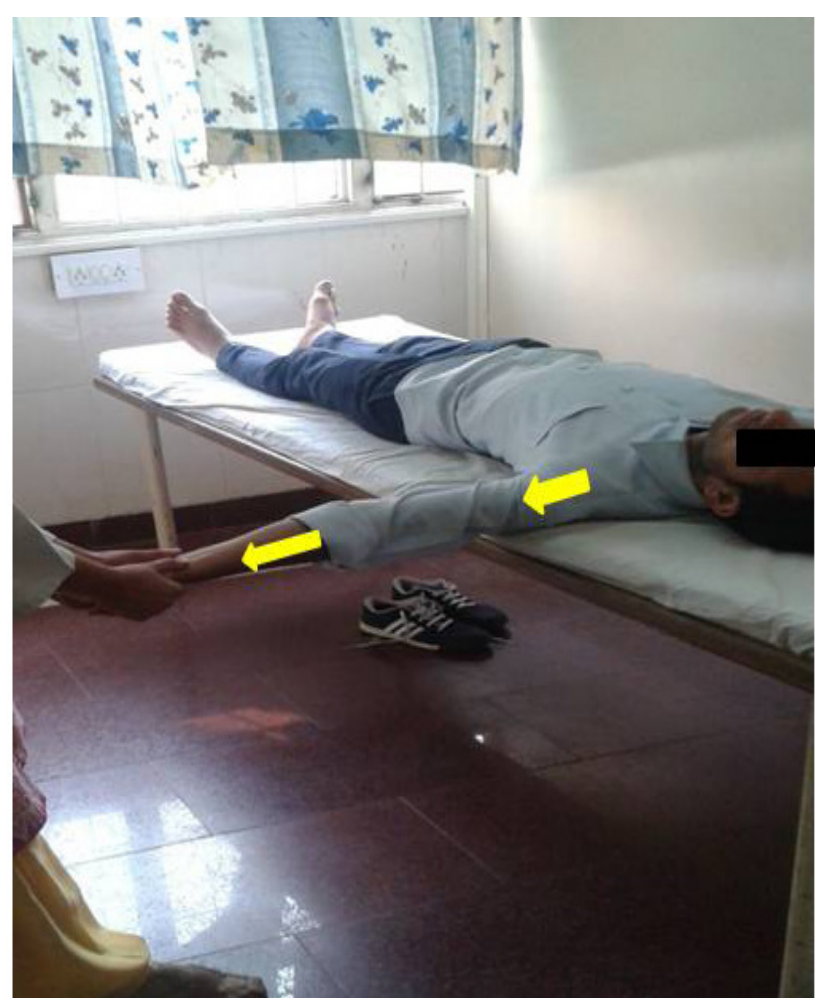

Figure 3 - With traction patient's forearm is supinated and slowly abducted to $90^{\circ}$

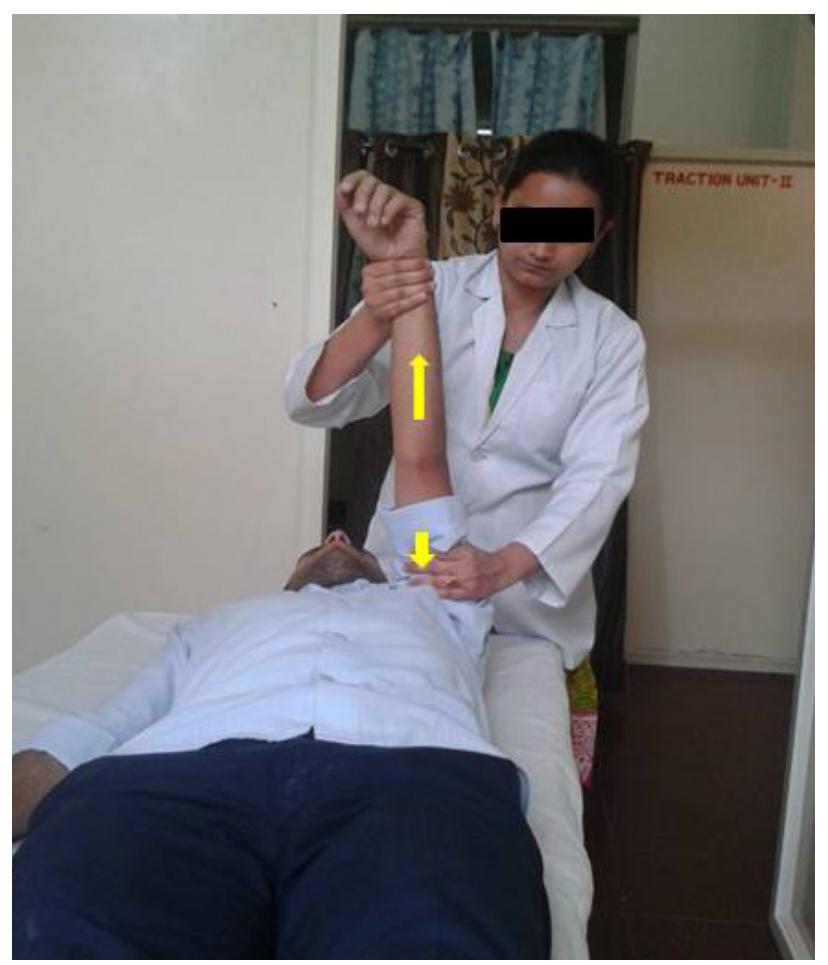

Figure 5 - Upward traction with shoulder flexion and scapula first protracted and then pushed against the couch for shoulder compression and scapular retraction 


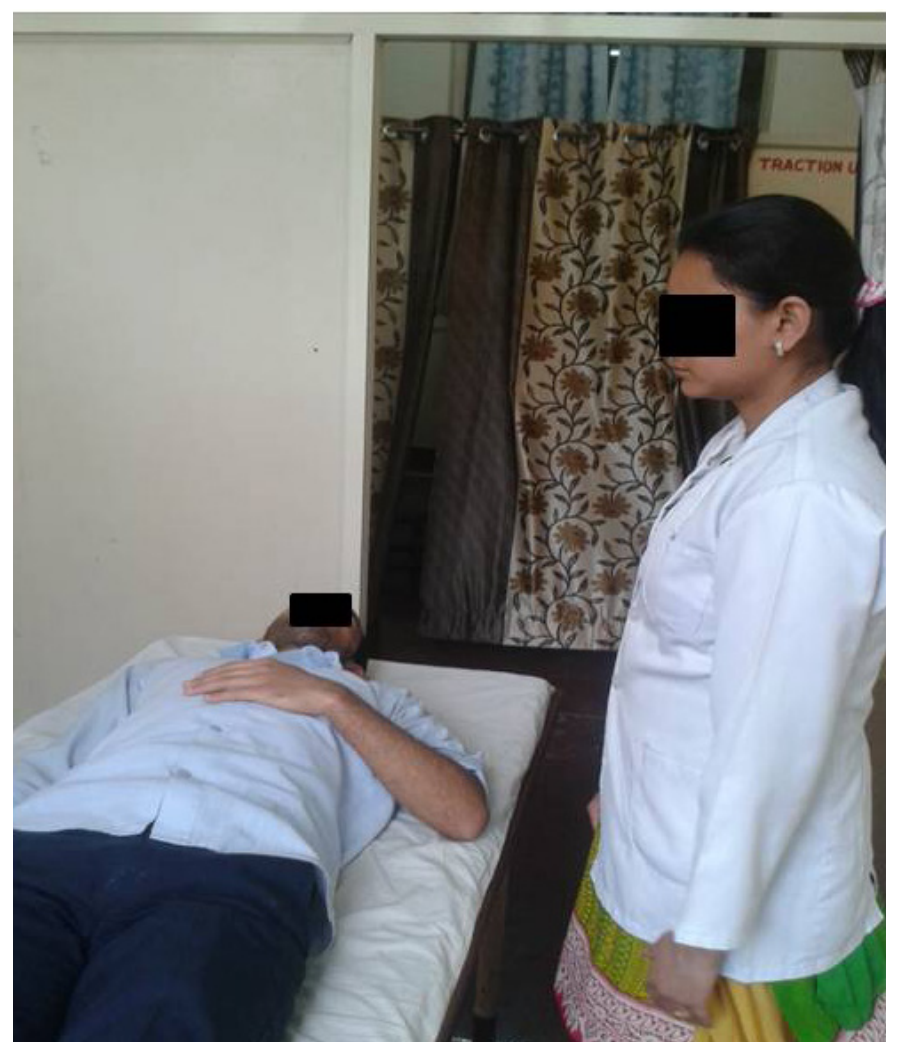

Figure 6 - Patient's arm returned to his side with elbow flexed and resting on his abdomen

In figure (2) and (3) with maintenance of traction the patient's forearm was first pronated then supinated and then his palms were stretched laterally and slightly downwards by using therapist's thenar eminence and fingers. Traction was maintained and his upper extremity was slowly abducted following pull into flexion and abduction was continued until his arm was abducted to $90^{\circ}$. In figure (4) there was continued slow horizontal abduction, flexion and rotation until the patient's arm was proximal to his ear. Therapist changed his handgrip and body position to move patient's arm into more flexion at shoulder joint and scapular protraction. In figure (5) the patient's shoulder is lifted off the treatment table at $90^{\circ}$ of flexion and scapular protraction, and then was pushed towards treatment table to compress shoulder joint and scapular retraction. After this the upward traction was maintained leading the scapula around the ribcage into full protraction while leading his arm into horizontal adduction. Slight elbow flexion with full functional horizontal adduction was allowed to achieve an end feel. Then patient's arm was returned to his side while maintaining traction, flexing his elbow and placing his hand in full contact with his abdomen as shown in figure (6). Gross MFR of upper trapezius in figure 7. 


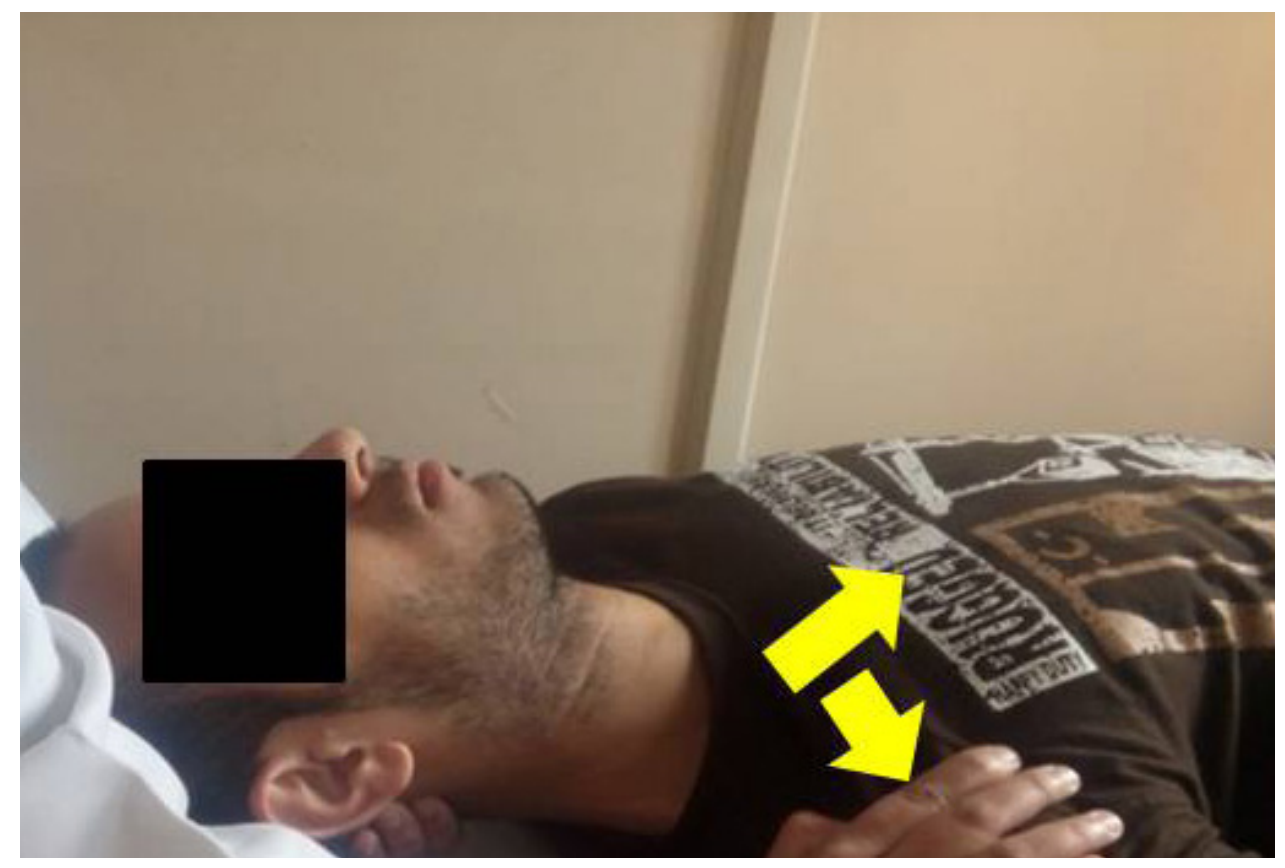

Figure 7 - Gross MFR of upper trapezius

\section{DATA ANALYSIS}

Data were analysed by with the Statistical Package for Social Sciences (SPSS, version 16. Inc., Chicago, IL) The normality of the data was checked by using Shapiro wilk test which showed that data was not normally distributed and hence the non parametric test, Wilcoxon Signed Rank test $(p<0.05)$ was used for analysing the data.

\section{RESULTS}

A total of 35 subjects were contacted for the study out of which 5 discontinued the treatment and 15 were excluded as they did not met the inclusion criteria and had other additional disorders along with CR. Demographic data of the participants recruited is displayed in table 1. Pre and post analyzed report of NDI, NPRS and ULNT1 are displayed in figures 8, 9 and 10 respectively. In all three outcome measures the statistical significant change of $p<0.001$ are observed.

Table 1 - Demographic characteristics of the individual with cervical radiculopathy (CR) recruited

(continued)

\begin{tabular}{ccccc}
\hline S. No. & AGE (IN YEARS) & Gender & HEIGHT (IN CM) & WEIGHT (IN KG) \\
\hline 1 & 30 & Male & 167 & 68 \\
2 & 28 & Female & 159 & 57 \\
3 & 35 & Female & 160 & 55 \\
4 & 40 & Male & 164 & 70 \\
5 & 39 & Female & 160 & 65 \\
6 & 42 & Male & 174 & 80 \\
7 & 37 & Female & 168 & 60
\end{tabular}


Table 1 - Demographic characteristics of the individual with cervical radiculopathy (CR) recruited

\begin{tabular}{ccccc}
\hline S. No. & AGE (IN YEARS) & Gender & HeIGHT (IN CM) & WeIGHT (IN KG) \\
\hline 8 & 39 & Male & 174 & 74 \\
9 & 43 & Female & 165 & 62 \\
10 & 33 & Female & 150 & 55 \\
11 & 36 & Male & 157 & 61 \\
12 & 31 & Female & 157 & 58 \\
13 & 42 & Male & 165 & 64 \\
14 & 30 & Male & 170 & 75 \\
15 & 34 & Female & 153 & 54 \\
\hline
\end{tabular}

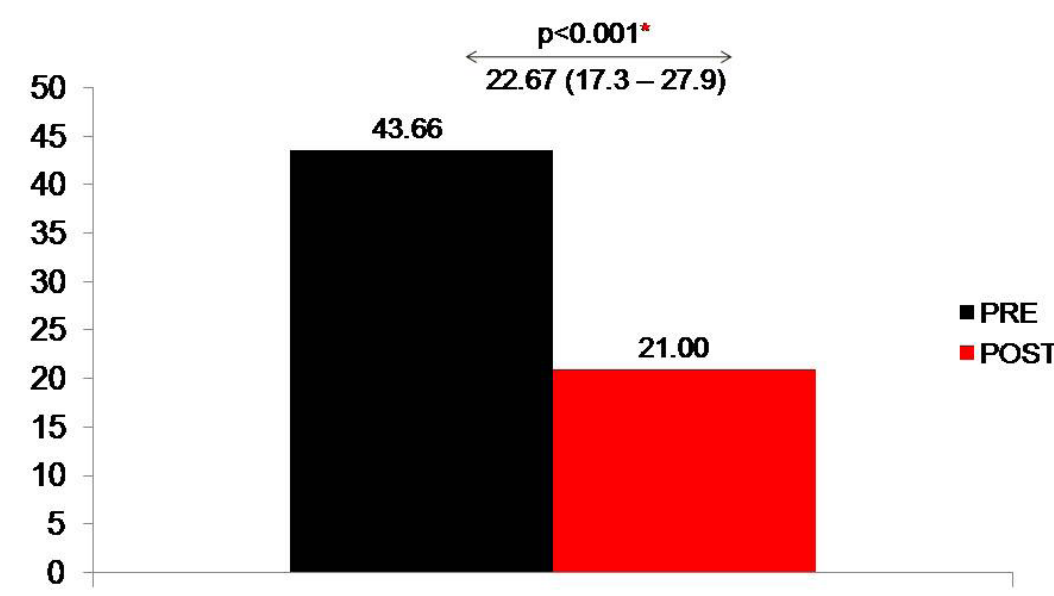

\section{NDI}

* Wikcoxon signed rank test

Figure 8 - Pre and post analysis of Neck Disability Index (NDI)

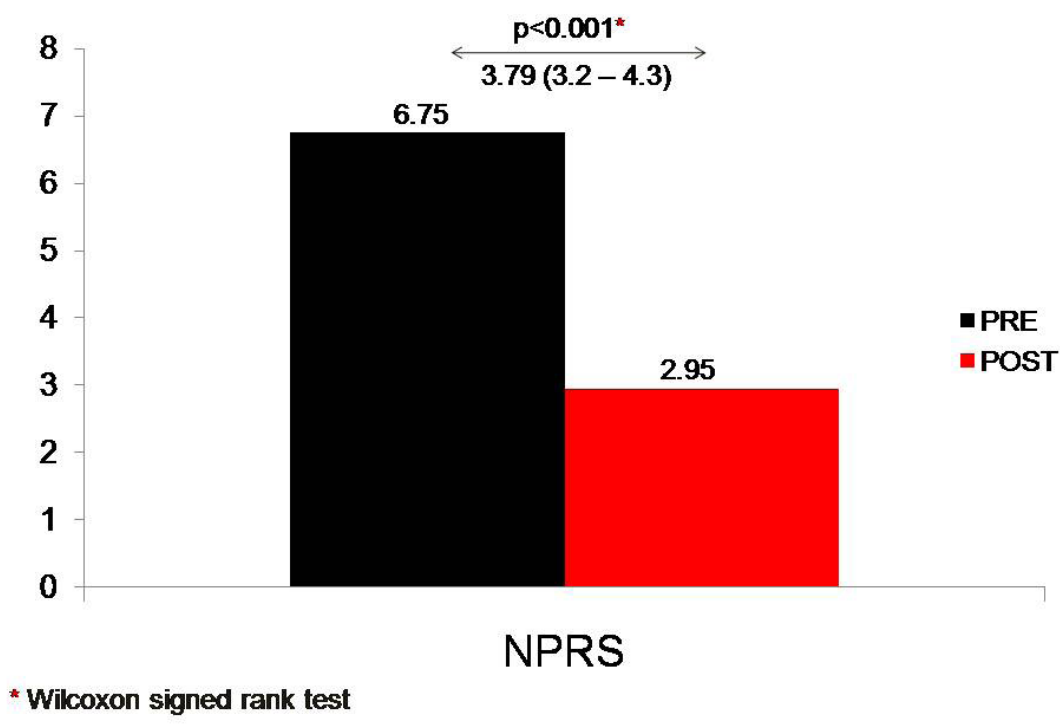

Figure 9 - Pre and post analysis of Numeric Pain Rating Scale (NPRS) 


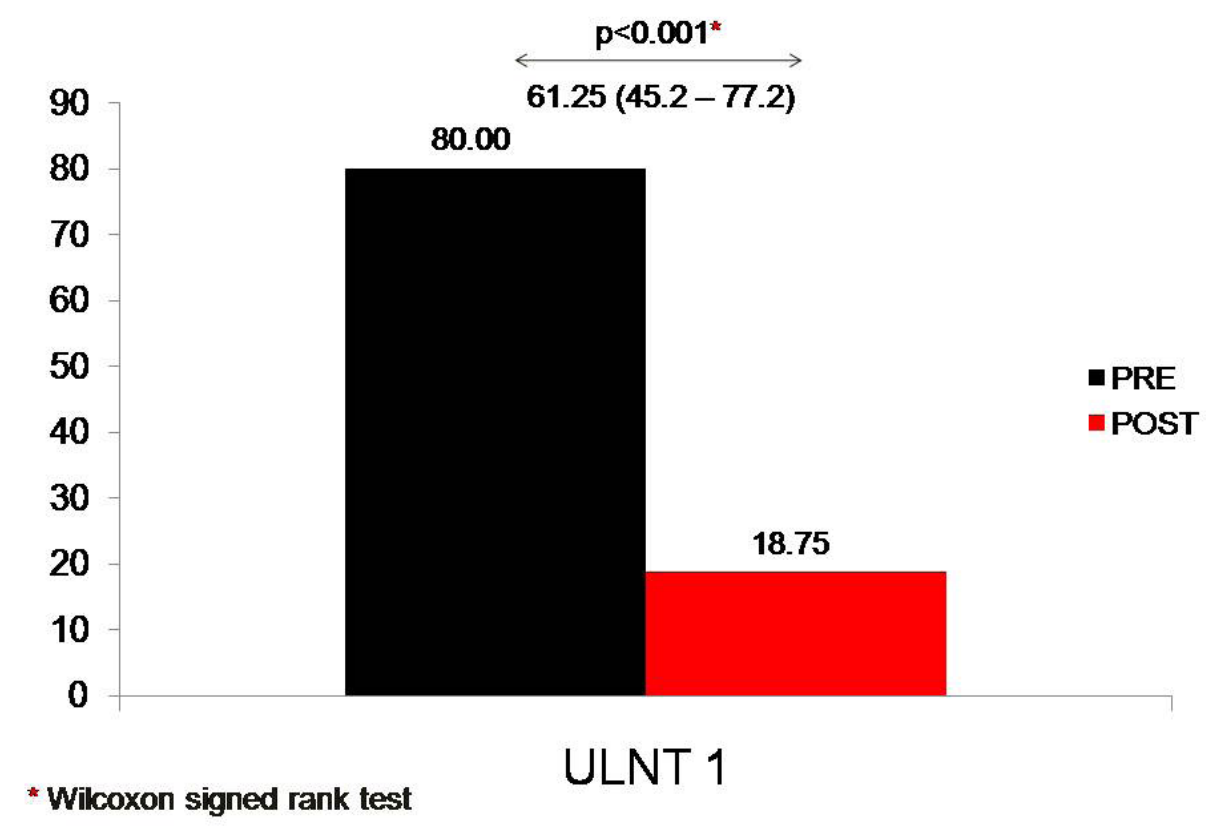

Figure 10 - Pre and post analysis of Upper Limb Neurodynamic Test for median nerve (ULNT 1)

\section{DISCUSSION}

This study included 15 patients with CR who were treated with MFR and showed significant improvement. MFR helps in improving the neck related arm pain in patients with CR. The study also supports the findings of a previous clinical trial performed by Nitsure et $a^{\left({ }^{(15)}\right.}$ on similar outcome measurements. The subjects were given an arm pull and gross stretch of posterior cervical musculature for 5 days. On commencement of the study, significant diminution in pain and improvement in disability was noticed.

The results are consistent with the case study reported by Alagha ${ }^{(21)}$ on a patient with low back pain and lumbar radiculopathy. The patient was treated by manipulation for 10 consecutive sessions followed by rehabilitation protocol of exercise therapy constituting advanced MFR therapy for another 12 sessions. After the above protocol patient reported a significant improvement in low back pain and radiculopathy. In addition, patient reported some improvement in balance also. The study shows that MFR can be used in treating the patients with radiculopathy.
In the study, overall situation of the subjects improved by the use of MFR as it is helpful in reducing muscle soreness, relieving joint stress, decreasing neuromuscular hypertonicity, increasing extensibility of musculotendinous junction, improving neuromuscular efficiency and correcting muscle imbalance along with maintenance of normal functional muscular length. ${ }^{(22)}$ MFR was found to reduce the neck disability and hence to help the patients to return to towards their previous normal life. The participants were convinced and motivated to not undergo any other therapeutic interventions besides the MFR as it is a hands on manual approach technique which could be proved more beneficial in decreasing disability and pain.

The major limitations of the study include small sample size and absence of control group. However, improvisation of the study can be done by comparison with the control group, giving adjunct treatment with MFR to the experimental group, pressure algometer should be used for the trigger points sensitivity and follow ups should be done. Future studies should be done with larger samples to overcome the above limitations. 


\section{CONCLUSION}

MFR reduces the neck disability and hence helps the patients to return to their previous normal life. MFR might be used as an effective treatment technique for the patients with $C R$.

\section{REFERENCES}

1. NG AT, Arora R, Arora L. Effectiveness of cervical traction on pain and disability in cervical radiculopathy. Int J Recent Sci Res. 2015;6(4):3609-11.

2. Boyles R, Toy P, Mellon J Jr, Hayes M, Hammer B. Effectiveness of manual physical therapy in the treatment of cervical radiculopathy: a systematic review. J Man Manip Ther. 2011;19(3):135-42.

3. Abbed KM, Coumans JV. Cervical radiculopathy: pathophysiology, presentation and clinical evaluation. Neurosurgery 2007;60(1 suppl):S28-34.

4. Thomes EJ, Scholten-Peters W, Koes B, Falla D, Verrhagen AP. The effectiveness of conservative treatment for patients with cervical radiculopathy: a systematic review. Clin J Pain. 2013;29(12):1073-86.

5. Fritz JM, Thackeray A, Brennan GP, Childs JD. Exercise only, exercise with mechanical traction, or exercise with over-door traction for patients with cervical radiculopathy, with or without consideration of status on a previously described subgrouping rule: $A$ randomized clinical trial. J Orthop Sports Phys Ther. 2014;44(2):45-57.

6. Rao R. Neck pain, cervical radiculopathy, and cervical myelopathy: pathophysiology, natural history, and clinical evaluation. J Bone Joint Surg Am. 2002;84(10)1872-81.

7. Olivero WC, Dulebohn SC. Results of halter cervical traction for the treatment of cervical radiculopathy: retrospective review of 81 patients. Neurosurg Focus 2002;12(2):1-5.

8. Sari H, Akarimak U, Uludag M. Active myofascial trigger points might be more frequent in patients with cervical radiculopathy. Eur J Phys Rehabil Med. 2012;48(2):237-44.

9. Letchuman R, Gay RE, Sherlerud RA, VanOstrand LA. Are tender points associated with cervical radiculopathy? Arch Phys Med Rehabil. 2005;86(7):1333-7.

10. Chu J, Schwartz I. eTioms Twitch relief method in chronic refractory myofascial pain(CRMP). Electromyogr. clin. Neurophysiol. 2008;48:31120.

11. Hsueh TC, Yu S, Khan TS, Hong CZ. Association of active myofascial trigger points and cervical disc lesions. J Formos Med Assoc. 1998;97(3):174-80.

12. Jeyanthi S, Arumugam N. Effectiveness of cranio-cervical training over myofascial pain syndrome: a case study. Int J Physiother Res. 2015;3(3):1032-36.

13. Hou CR, Tsai LC, Cheng KF, Chung KC, Hong CZ. Immediate effects of various physical therapeutic modalities on cervical myofascial pain and trigger point sensitivity. Arch Phys Med Rehabil. 2MOO2;83:1406-14.

14. Jay S. A comparative study between muscle energy technique and myofascial release therapy on myofascial trigger points in upper fibers of trapezius. Indian J. Physiother Occup Ther. 2012;6(3):144-8.

15. Nitsure P, Welling A. Effect of gross myofascial release of upper limb and neck on pain and function in subjects with mechanical neck pain with upper limb radiculopathy: A clinical trial. Int J Dental Med Res. 2014;1(3):8-16.

16. F SS, Manhas A, Parekh K. The effect of upper limb tension test in the management of ROM limitation and pain in cervical radiculopathy. Int J Physiother Res. 2015;3(3):1065-7.

17. Woods BI, Hilibrand AS. Cervical radiculopathy: epidemiology, etiology, diagnosis and treatment. J Spinal Disord Tech. 2O15;28(5):E251-9.

18. Moustafa IM, Diab AA. Multimodal treatment program comparing 2 different traction approaches for patients with discogenic cervical radiculopathy: a randomized controlled trial. J Chiropr Med. 2014;13:157-67.

19. Langevin P, Desmeules F, Lamothe M, Robitaille S, Roy JS. Comparison of 2 manual therapy and 
exercise protocols for cervical radiculopathy: a randomized clinical trial evaluating short-term effects. J Orthop Sports Phys Ther. 2015;45(1):417.

2O. Sambyal S, Kumar S. Comparison between nerve mobilization and conventional physiotherapy in patients with cervical radiculopathy. Int J Innov Res Dev. 2013;2(8):442-5.
21. Alagha B. Chiropractic and rehabilitation management of a patient with extraforaminal entrapment of $L 4$ nerve with balance problem. J Back Musculoskelet Rehabil. 2015;28(3):603-7.

22. Shah S, Bhalara A. Myofascial release. Int J Health Sci Res. 2O12;2(2):69-77. 